PROCEEDINGS OF THE

AMERICAN MATHEMATICAL SOCIETY

Volume 133, Number 5, Pages 1343-1347

S 0002-9939(04)07811-6

Article electronically published on December 15, 2004

\title{
RIESZ POINTS OF UPPER TRIANGULAR OPERATOR MATRICES
}

\author{
BRUCE A. BARNES
}

(Communicated by Joseph A. Ball)

\begin{abstract}
Two results are proved which concern Riesz points of upper triangular operator matrices. Applications are made to questions involving when Weyl's Theorem holds for an upper triangular operator matrix.
\end{abstract}

\section{INTRODUCTION}

Let $T \in B(X)$, the algebra of all bounded linear operators on a Banach space $X$. Let $\mathbf{N}(T)$ denote the null space of $T$, and let $\sigma(T)$ denote the usual operator spectrum of $T$.

A number $\lambda_{0} \in \mathbf{C}$ is a Riesz point of $T$ if either $\lambda_{0} \notin \sigma(T)$ or $\lambda_{0}$ is an isolated point of $\sigma(T)$ and the spectral projection corresponding to the set $\left\{\lambda_{0}\right\}$ (defined as usual using the holomorphic functional calculus) has finite-dimensional range.

Let $\mathbf{R Z}(T)$ be the set of all Riesz points of $T$ which are in $\sigma(T)$. There are a number of useful characterizations of Riesz points of an operator; see [B1. Proposition 2]. Here is one such characterization: Let $\Phi^{0}$ be the set of all Fredholm operators in $B(X)$ which have index zero.

$\lambda_{0} \in \mathbf{R Z}(T)$ if and only if $\lambda_{0}$ is an isolated point of $\sigma(T)$ and $\left(\lambda_{0}-T\right) \in \Phi^{0}$.

Two important essential spectra of $T \in B(X)$ are:

the Weyl spectrum of $T, \sigma_{W}(T)=\left\{\lambda \in \mathbf{C}:(\lambda-T) \notin \Phi^{0}\right\}$;

the Browder spectrum of $T, \sigma_{B}(T)=\{\lambda \in \mathbf{C}: \lambda$ is not a Riesz point of $T\}$.

Clearly, $\sigma_{W}(T) \subseteq \sigma_{B}(T) \subseteq \sigma(T)$. Now define $\Pi_{00}(T)$ to be the set of all isolated points $\lambda_{0}$ of $\sigma(T)$ such that $\mathbf{N}\left(\lambda_{0}-T\right)$ is finite dimensional. It is easy to check that $\mathbf{R Z}(T) \subseteq \Pi_{00}(T)$. Here are two interesting spectral properties which an operator $T \in B(X)$ might have:

Weyl's Theorem holds for $T$ if $\sigma_{W}(T)=\sigma(T) \backslash \Pi_{00}(T)$;

Browder's Theorem holds for $T$ if $\sigma_{W}(T)=\sigma(T) \backslash \mathbf{R Z}(T)$.

In fact, Browder's Theorem holds for $T$ exactly when $\sigma_{W}(T)=\sigma_{B}(T)$. Clearly, if Browder's Theorem holds for $T$, and $\mathbf{R Z}(T)=\Pi_{00}(T)$, then Weyl's Theorem holds for $T$. The converse of this statement is also true; see [B1, Corollary 5].

Received by the editors November 4, 2003.

2000 Mathematics Subject Classification. Primary 47A10.

Key words and phrases. Riesz point, upper triangular operator matrix, Weyl's Theorem.

(C)2004 American Mathematical Society Reverts to public domain 28 years from publication 
In [B1], Browder's Theorem is called, "Version II of Weyl's Theorem". Additional information concerning Browder's Theorem and Weyl's Theorem for bounded linear operators can be found in [HL].

In this paper, we first prove two results concerning Riesz points of an upper triangular operator matrix. Then we consider questions concerning Browder's Theorem and Weyl's Theorem in this same setting. This work is motivated by the recent papers on spectral properties of upper triangular operator matrices $[\mathrm{BB}$, $[\mathrm{DH}]$, and $[\mathrm{L}]$. The author's paper, [B2], deals with the same topic.

\section{RIESZ POINTS OF AN UPPER TRIANGULAR OPERATOR MATRIX}

Let $X_{k}, 1 \leq k \leq n$, be Banach spaces, and let $X=X_{1} \oplus \ldots \oplus X_{n}$. Assume for $1 \leq j, k \leq n$ that $T_{j k}$ is a bounded linear operator defined on $X_{k}$ with values in $X_{j}$. Then the $n$ by $n$ operator matrix $T=\left(T_{j k}\right)$ defines a linear operator in $B(X)$ in the usual way. $T=\left(T_{j k}\right) \in U_{n}$, the algebra of all upper triangular operator matrices, when $T_{j k}=0$ whenever $j>k$. For $T=\left(T_{j k}\right)$, we denote the diagonal operator matrix with entries $T_{k k}$ down the diagonal by $d g(T)=\left(T_{11}, \ldots, T_{n n}\right)$.

In this section we consider Riesz points of operator matrices in $U_{n}$. We need the following elementary fact.

Note. Assume that $P=P^{2}$ and $P=F+N$ where $F$ has finite-dimensional range and $N^{m}=0$ for some positive integer $m$. Then $P$ has finite-dimensional range.

Proof. $P=P^{2}=(F+N)(F+N)=F^{2}+N F+F N+N^{2}=F_{1}+N^{2}$, where $F_{1}$ has finite-dimensional range. Using this argument repeatedly, $P=F_{k}+N^{2^{k}}$ where $F_{k}$ has finite-dimensional range for $k \geq 1$. Since $N$ is nilpotent, $P=F_{k}$ for $k$ sufficiently large.

For $T=\left(T_{j k}\right) \in U_{n}$, the spectrum of $T$ relative to the Banach algebra $U_{n}$ is $\bigcup_{k=1}^{n} \sigma\left(T_{k k}\right)$ [B2, Proposition 1.1]. One practical effect of this is that when $\lambda \notin \bigcup_{k=1}^{n} \sigma\left(T_{k k}\right)$, then $(\lambda-T)^{-1} \in U_{n}$. Also note that $T \rightarrow d g(T)$ is an algebra homomorphism of $U_{n}$ onto the algebra of diagonal operator matrices. Suppose $T=\left(T_{j k}\right) \in U_{n}$ has an inverse $T^{-1}=S=\left(S_{j k}\right) \in U_{n}$. Then $I=$ $d g(S T)=d g(S) d g(T)$. It follows that $S_{k k}=\left(T_{k k}\right)^{-1}$ for all $k$, that is, $d g\left(T^{-1}\right)=$ $\left(T_{11}^{-1}, T_{22}^{-1}, \ldots, T_{n n}^{-1}\right)$. We use these facts in the proof of the next theorem.

For $\lambda_{0} \in \mathbf{C}$ and $r>0$, let $D\left(\lambda_{0} ; r\right)=\left\{\lambda \in \mathbf{C}:\left|\lambda-\lambda_{0}\right|<r\right\}$.

Theorem 1. Assume $T=\left(T_{j k}\right) \in U_{n}$. If $\lambda_{0}$ is a Riesz point of $T_{k k}$ for $1 \leq k \leq n$, then $\lambda_{0}$ is a Riesz point of $T$.

Proof. Since $\lambda_{0}$ is a Riesz point of $T_{k k}$ for all $k$, we can choose $r>0$ such that $D\left(\lambda_{0} ; r\right) \backslash\left\{\lambda_{0}\right\} \subseteq \sigma\left(T_{k k}\right)^{c}$ for $1 \leq k \leq n$. Therefore, $D\left(\lambda_{0} ; r\right) \backslash\left\{\lambda_{0}\right\} \subseteq$ $\left[\bigcup_{k=1}^{n} \sigma\left(T_{k k}\right)\right]^{c}$. Let $\gamma(t)=\lambda_{0}+\left(\frac{r}{2}\right) e^{i t}, t \in[0,2 \pi]$. For any $\lambda$ in the image of $\gamma$, $(\lambda-T)^{-1}$ exists, is in $U_{n}$, and $d g\left((\lambda-T)^{-1}\right)=\left(\left(\lambda-T_{11}\right)^{-1},\left(\lambda-T_{22}\right)^{-1}, \ldots\right.$, $\left.\left(\lambda-T_{n n}\right)^{-1}\right)$. Let $P_{0}$ be the spectral projection $P_{0}=\frac{1}{2 \pi i} \int_{\gamma}(\lambda-T)^{-1} d \lambda \in U_{n}$. Note that $d g\left(P_{0}\right)=\left(P_{1}, P_{2}, \ldots, P_{n}\right)$ where $P_{k}=\frac{1}{2 \pi i} \int_{\gamma}\left(\lambda-T_{k k}\right)^{-1} d \lambda$ for $1 \leq k \leq n$.

Since $\lambda_{0}$ is a Riesz point of $T_{k k}$ for all $k, P_{k}$ has finite-dimensional range for all $k$. Thus, $d g\left(P_{0}\right)$ has finite-dimensional range, and $P_{0}=d g\left(P_{0}\right)+\left(P_{0}-d g\left(P_{0}\right)\right)$ where $\left(P_{0}-d g\left(P_{0}\right)\right)^{n}=0$. It follows from the Note above that $P_{0}$ has finitedimensional range. This proves that $\lambda_{0}$ is a Riesz point of $T$. 
Theorem 2. Assume $T=\left(T_{j k}\right) \in U_{n}$ and $\sigma(T)=\bigcup_{k=1}^{n} \sigma\left(T_{k k}\right)$. If $\lambda_{0}$ is a Riesz point of $T$, then $\lambda_{0}$ is a Riesz point of $T_{k k}$ for $1 \leq k \leq n$.

Proof. Assume that $\lambda_{0}$ is a Riesz point of $T$. If $\lambda_{0}-T$ is invertible, then $\lambda_{0}-T_{k k}$ is invertible for $1 \leq k \leq n$ by hypothesis. Now assume that $\lambda_{0} \in \sigma(T)$, so $\lambda_{0}$ is an isolated point of $\sigma(T)$. Then for all $k$, either $\lambda_{0}$ is an isolated point of $\sigma\left(T_{k k}\right)$ or $\lambda_{0} \notin \sigma\left(T_{k k}\right)$. Just as in the proof of the previous result, we can choose $\gamma$ and compute the spectral projections $P_{0}=\frac{1}{2 \pi i} \int_{\gamma}(\lambda-T)^{-1} d \lambda$ and $P_{k}=\frac{1}{2 \pi i} \int_{\gamma}\left(\lambda-T_{k k}\right)^{-1} d \lambda$ for all $k$ (when $\lambda_{0} \notin \sigma\left(T_{k k}\right)$, then $P_{k}=0$ ). Again, $d g\left(P_{0}\right)=\left(P_{1}, P_{2}, \ldots, P_{n}\right)$, and since $P_{0}$ has finite-dimensional range, each $P_{k}$ has finite-dimensional range. Therefore, $\lambda_{0}$ is a Riesz point of $T_{k k}$ for all $k$.

\section{WEYL'S THEOREM FOR UPPER TRIANGULAR OPERATOR MATRICES}

In this section we prove two results concerning Weyl's Theorem for upper triangular operator matrices. First we prove a preliminary result.

Proposition 3. Assume $T=\left(T_{j k}\right) \in U_{n}$. If $\sigma_{W}(T)=\bigcup_{k=1}^{n} \sigma_{W}\left(T_{k k}\right)$, then $\sigma(T)=\bigcup_{k=1}^{n} \sigma\left(T_{k k}\right)$.

Proof. It is always true that $\sigma(T) \subseteq \bigcup_{k=1}^{n} \sigma\left(T_{k k}\right)$. Now suppose that $\lambda_{0} \notin \sigma(T)$, so $\lambda_{0}-T$ is invertible. Since $\lambda_{0}-T \in \Phi^{0}$, we have by hypothesis that $\lambda_{0}-T_{k k} \in \Phi^{0}$ for $1 \leq k \leq n$. Then from the proof of Theorem 4.1 in [B2], it follows that $\left(\lambda_{0}-T\right)^{-1} \in U_{n}$. This implies that $\left(\lambda_{0}-T_{k k}\right)^{-1}$ exists for $1 \leq k \leq n$. Thus, $\lambda_{0} \notin \bigcup_{k=1}^{n} \sigma\left(T_{k k}\right)$. This proves the proposition.

For any operator $T$, the sets $\sigma_{W}(T)$ and $\mathbf{R Z}(T)$ are disjoint, since when $\lambda_{0} \in \mathbf{R Z}(T),\left(\lambda_{0}-T\right) \in \Phi^{0}$. It follows easily from this that the equality $\sigma_{W}(T)=$ $\sigma(T) \backslash \mathbf{R Z}(T)$ is equivalent to $\sigma(T)=\sigma_{W}(T) \cup \mathbf{R Z}(T)$. Thus, this last equality is another equivalent way of asserting that $T$ satisfies Browder's Theorem.

Theorem 4. Assume $T=\left(T_{j k}\right) \in U_{n}$. Assume that $T$ satisfies:

(a) $\sigma_{W}(T)=\bigcup_{k=1}^{n} \sigma_{W}\left(T_{k k}\right)$;

(b) for each $k, \quad \sigma\left(T_{k k}\right)=\sigma_{W}\left(T_{k k}\right) \cup \mathbf{R Z}\left(T_{k k}\right)$;

(c) for each $k$, every isolated point of $\sigma\left(T_{k k}\right)$ is a Riesz point.

Then $\sigma(T)=\sigma_{W}(T) \cup \mathbf{R Z}(T)$ and every isolated point of $\sigma(T)$ is a Riesz point. Also, $T$ satisfies Weyl's Theorem.

Proof. Assume that $\lambda_{0}$ is an isolated point of $\sigma(T)$. By (a) and Proposition 3, we have $\sigma(T)=\bigcup_{k=1}^{n} \sigma\left(T_{k k}\right)$. Thus for all $k$, either $\lambda_{0} \notin \sigma\left(T_{k k}\right)$ or $\lambda_{0}$ is an isolated point of $\sigma\left(T_{k k}\right)$. By (c), $\lambda_{0}$ is a Riesz point of $T_{k k}$ for all $k$. Then from Theorem 1 it follows that $\lambda_{0}$ is a Riesz point of $T$.

Now suppose that $\lambda_{0} \notin \sigma_{W}(T)$, so $\lambda_{0}-T \in \Phi^{0}$. Then from (a), $\lambda_{0}-T_{k k} \in \Phi^{0}$ for $1 \leq k \leq n$. Therefore from (b), for all $k, \lambda_{0}$ is a Riesz point of $T_{k k}$. It follows from Theorem 1 that $\lambda_{0}$ is a Riesz point of $T$.

We have proved above that $\sigma_{W}(T)=\sigma_{B}(T)$. Now we show $\Pi_{00}(T)=\mathbf{R Z}(T)$. The inclusion, $\mathbf{R Z}(T) \subseteq \Pi_{00}(T)$, always holds. Suppose $\lambda_{0} \in \Pi_{00}(T)$. Then $\lambda_{0}$ is an isolated point of $\sigma(T)$. But then as shown above, $\lambda_{0}$ is a Riesz point of $T$. Therefore, $\Pi_{00}(T)=\mathbf{R Z}(T)$. This proves that Weyl's Theorem holds for $T$.

With reference to Theorem 4, we give an example of one type of problem which can occur when some isolated points are not Riesz points. 
Assume that $Q$ is quasinilpotent in $B(X)$ and that $\mathbf{N}(Q)=\{0\} \quad$ (some weighted shifts have these properties). Let $P$ be a projection operator on $X$ with one-dimensional range. Let $T$ be the diagonal operator, $T=\left(\begin{array}{cc}I+Q & 0 \\ 0 & P\end{array}\right)$. We list some spectral properties of the operators involved:

(1) $\sigma(I+Q)=\{1\} ; \sigma(P)=\{0,1\} ; \sigma(T)=\{0,1\}$.

(2) $1 \in \Pi_{00}(T)$.

Proof. Assume that $x_{0} \in X$ and that the range of $P$ is the set $\left\{\lambda x_{0}: \lambda \in \mathbf{C}\right\}$. Then $T\left(\begin{array}{c}0 \\ x_{0}\end{array}\right)=\left(\begin{array}{c}0 \\ x_{0}\end{array}\right)$. Also, if $T\left(\begin{array}{c}x_{1} \\ x_{2}\end{array}\right)=\left(\begin{array}{c}x_{1} \\ x_{2}\end{array}\right)$, then $(I+Q) x_{1}=x_{1}$, so $Q x_{1}=0$, and therefore, $x_{1}=0$. Also, $P x_{2}=x_{2}$, so $x_{2}$ is a scalar multiple of $x_{0}$. This verifies that $N(I-T)=\left\{\left(\begin{array}{c}0 \\ \lambda x_{0}\end{array}\right): \lambda \in \mathbf{C}\right\}$.

(3) $1 \notin \mathbf{R Z}(T)$ [since $1 \notin \mathbf{R Z}(I+Q)]$.

(4) $\sigma_{W}(P)=\{0\}=\sigma_{B}(P)$ [note that $\left.I-P \in \Phi^{0}\right] ; \sigma_{W}(I+Q)=\{1\}=$ $\sigma_{B}(I+Q) ; \sigma_{W}(T)=\{0,1\}=\sigma_{W}(P) \cup \sigma_{W}(I+Q)$.

(5) $\sigma_{W}(T)=\{0,1\}=\sigma_{B}(T)$.

(6) $T$ does not satisfy Weyl's Theorem, since $\sigma_{W}(T)=\{0,1\}$, while $\sigma(T) \backslash \Pi_{00}(T)$ $=\{0\}$. Both $I+Q$ and $P$ do satisfy Weyl's Theorem.

Let $K(X)$ be the space of all compact linear operators on $X$. The following fact is known.

Fact. For any $T \in B(X)$, there exists $J \in K(X)$ such that $\sigma_{W}(T+J)=$ $\sigma_{B}(T+J)$.

This fact follows from part of the proof of Corollary $\mathrm{C}^{*} .2 .6$, p. 76 in [BMSW]. The proof is set in a $\mathrm{C}^{*}$-algebra, but it works for $B(X)$. Now we look at a similar result involving Weyl's Theorem. Specifically, we consider the question:

What conditions on an operator $T \in B(X)$ imply the existence of an operator $J \in K(X)$ such that $T+J$ satisfies Weyl's Theorem?

Given a set of $n$ operators $\left\{J_{1}, J_{2}, \ldots, J_{n}\right\}$, let $d g\left(J_{1}, J_{2}, \ldots, J_{n}\right)$ be the diagonal operator matrix with entries $J_{1}, \ldots, J_{n}$ down the main diagonal.

Theorem 5. (1) Assume that $T \in B(X)$ and that $\sigma_{W}(T)$ has no isolated points. Then there exists $J \in K(X)$ such that $T+J$ satisfies Weyl's Theorem.

(2) Assume that $T=\left(T_{j k}\right) \in U_{n}$ and that $T$ satisfies:

(i) $\sigma_{W}(T)=\bigcup_{k=1}^{n} \sigma_{W}\left(T_{k k}\right) ; \quad$ and (ii) for all $k, \sigma_{W}\left(T_{k k}\right)$ has no isolated points.

Then there exists $J=d g\left(J_{1}, J_{2}, \ldots, J_{n}\right)$, where each $J_{k}$ is compact, such that $T+J$ satisfies Weyl's Theorem.

Proof. (1): By the Fact, there exists $J \in K(X)$ such that $T+J$ has the property $\sigma_{W}(T+J)=\sigma_{B}(T+J)$. Now it always holds that $\mathbf{R Z}(T+J) \subseteq \Pi_{00}(T+J)$. Assume $\quad \lambda_{0} \in \Pi_{00}(T+J)$. Since $\sigma_{W}(T+J)=\sigma_{W}(T)$ has no isolated points, $\lambda_{0} \notin \sigma_{W}(T+J)$. Then $\lambda_{0}-(T+J) \in \Phi^{0}$, so $\lambda_{0}$ is a Riesz point of $T+J$. This proves $\mathbf{R Z}(T+J)=\Pi_{00}(T+J)$. It follows that $T+J$ satisfies Weyl's Theorem.

(2): Assume the hypotheses in (2). By (1), for each $k$ there exists a compact operator $J_{k}$ such that $T_{k k}+J_{k}$ satisfies Weyl's Theorem. Let $J=d g\left(J_{1}, J_{2}, \ldots, J_{n}\right)$. We verify that $T+J$ satisfies hypotheses (a), (b) and (c) of Theorem 4. First, by (i), $\sigma_{W}(T+J)=\bigcup_{k=1}^{n} \sigma_{W}\left(T_{k k}+J_{k}\right)$, so (a) holds. By the choice of the operators $J_{k}$, (b) in the statement of Theorem 4 holds: $\sigma\left(T_{k k}+J_{k}\right)=\sigma_{W}\left(T_{k k}+J_{k}\right) \cup \mathbf{R Z}\left(T_{k k}+J_{k}\right)$. 
By (ii), $\sigma_{W}\left(T_{k k}+J_{k}\right)$ has no isolated points. This together with $\sigma\left(T_{k k}+J_{k}\right)=$ $\sigma_{W}\left(T_{k k}+J_{k}\right) \cup \mathbf{R Z}\left(T_{k k}+J_{k}\right)$ implies that every isolated point of $\sigma\left(T_{k k}+J_{k}\right)$ is a Riesz point of $T_{k k}+J_{k}$. This verifies (c) of Theorem 4. Thus, Theorem 4 applies to $T+J$, so $T+J$ satisfies Weyl's Theorem.

In regard to Theorem 5, we note a simple example of an operator $T$ such that $\sigma(T)$ has no isolated points, but $\sigma_{W}(T)$ has an isolated point. Let $\left\{e_{k}: k \geq 1\right\}$ be the usual Hilbert space basis of $\ell^{2}$. Let $X=\ell^{2} \oplus \ell^{2} \oplus \ell^{2}$, and set $T=d g(S, B, P)$, where $S$ is the unilateral shift, $B$ is the unilateral backward shift, and $P$ is the orthogonal projection on $\ell^{2}$ with range the closed linear span of $\left\{e_{2 k}: k \geq 1\right\}$. Then it is not difficult to check that $\sigma(T)=\{\lambda:|\lambda| \leq 1\}$, while $\sigma_{W}(T)=\{\lambda:|\lambda|=1\} \cup\{0\}$.

\section{REFERENCES}

[B1] B. Barnes, Riesz points and Weyl's Theorem, Integral Equ. and Operator Theory 34 (1999), 187-196. MR1694707 (2000d:47006)

[B2] B. Barnes, The spectral theory of upper triangular matrices with entries in a Banach algebra, Math. Nachr. 241 (2002), 5-20. MR1912373 (2003e:47004)

[BMSW] B. Barnes, G. Murphy, R. Smyth, and T.T. West, Riesz and Fredholm Theory in Banach Algebras, Pitman, Boston-London-Melbourne, 1982. MR0668516 (84a:46108)

[BB] M. Barraa and M. Boumazgour, A note on the spectrum of an upper triangular operator matrix, Proc. Amer. Math. Soc. 131 (2003), 3083-3088. MR1993217|(2004d:47009)

[DH] S. Djordjevic and Y. Han, A note on Weyl's Theorem for operator matrices, Proc. Amer. Math. Soc. 131 (2002), 2543-2547. MR1974653 (2004a:47002)

[HL] R. Harte and W. Lee, Another note on Weyl's Theorem, Trans. Amer. Math. Soc. 349 (1997), 2115-2124. MR 1407492(98j:47024)

[L] W. Lee, Weyl's spectra of operator matrices, Proc. Amer. Math. Soc. 129 (2000), 131138. MR1784020 (2001f:47003)

Department of Mathematics, University of Oregon, Eugene, Oregon 97403

E-mail address: barnes@math.uoregon.edu 